\title{
La dimensión terapéutica del espacio relacional público.
}

The therapeutic dimension of public relational space.

\author{
José Ramón Boxó Cifuentes a, Joaquín Aragón Ortega ${ }^{\text {b }}$, Orlando Benito Riesco ${ }^{\text {, }}$, Leonor Ruiz \\ Sicilia ${ }^{\mathrm{d}}$, Miguel Ángel Rubio González ${ }^{\mathrm{e}}$. \\ ${ }^{a}$ Terapeuta y médico de familia. ${ }^{b}$ Terapeuta de familia, enfermero especialista en salud mental y antropólo- \\ go social. ${ }^{\circ}$ Terapeuta de familia y psicólogo clínico. ${ }^{d}$ Terapeuta de familia y psiquiatra. ${ }^{e}$ Terapeuta de fami- \\ lia y enfermero especialista en salud mental. ${ }^{a, b, c, d y e}$ Programa de terapia familiar. Hospital Universitario \\ Virgen de la Victoria. Málaga, España.
}

Correspondencia: José Ramón Boxó Cifuentes (terapiafamiliarmalaga@gmail.com)

Recibido: 08/04/2013; aceptado con modificaciones: 03/11/2013

\begin{abstract}
RESUMEN: Presentamos un modelo de trabajo en terapia familiar organizado en torno a la representación por parte del equipo de profesionales de los componentes del espacio público de comunicación interpersonal. Analizamos los componentes conceptuales de los espacios privado y público y el trabajo práctico con ellos basado en la adaptación del equipo terapéutico a las fases evolutivas de la familia durante el proceso de la terapia, que permite definir un itinerario guía en su progreso hasta su conclusión.

PALABRAS CLAVE: Privacidad, Relación con lo público, Terapia familiar, Comunicación, Relaciones interpersonales, Terapia narrativa.
\end{abstract}

\begin{abstract}
We present a working model in family therapy organized around the representation by the team of professionals of public space components of interpersonal communication. We analyze the conceptual components of private and public spaces and practical work with them based on the adaptation of the therapeutic team to the developmental stages of the family during the therapy process, which allows to define a itinerary guide in his progress up to his conclusion. KEY WORDS: Privacy, Public relations, Family therapy, Communication, Interpersonal relations, Narrative therapy.
\end{abstract}

\section{Introducción}

El desafío que supone para un equipo de terapia familiar la solicitud de ayuda por parte de los consultantes y los profesionales que los derivan, hace pertinente una reflexión en torno al modo en que se organiza la atención y los fundamentos sobre los que reposa la racionalidad de la ayuda y su aplicación.

El proceso parece iniciarse con una disposición a escuchar. La mera acción de escuchar, pone en marcha una pluralidad de poderosos fenómenos con repercusiones sanadoras en el sentido de resolución de conflictividad y sufrimiento evitable.

En un trabajo preliminar (1) proponíamos cuatro aspectos fenomenológicos de la disposición a escuchar que podrían hacer de ella una acción terapéutica teniendo en cuenta las correlaciones subyacentes que le atribuimos: 
1. Migración comunicacional desde el espacio privado de relación al espacio público: enfrenta la patología de la puntuación de los hechos

2. Apertura de la reflexividad y recuperación del sentido común: enfrenta la distorsión de la percepción del mundo

3. Validación del self: enfrenta la crisis de identidad

4. Estructura narrativa: enfrenta la historia saturada por el problema

En este trabajo pretendemos centrarnos en la dimensión terapéutica que supone la toma de decisión por parte de la familia de sacar su conflictividad privada a una esfera de análisis configurada por lo público. Tras una revisión de la literatura pertinente seleccionamos aquellos conceptos y aportaciones de otros autores que se han mostrado relevantes para la creación de este texto; también recogemos datos de nuestra actividad como equipo de terapia familiar en un modelo público de salud bajo un paradigma sistémico evolucionado que incorpora elementos de la teoría de la acción comunicativa y de la teoría del reconocimiento (2). Para una mejor comprensión remitimos al lector a dos textos elaborados por los mismos autores y que han sido referentes en el proceso de construcción del presente artículo (3-4).

Circunscribimos el espacio público al encuentro propiciado por el sistema de salud, donde familias con dificultades tienen oportunidad de acceder para el diálogo facilitado por un equipo profesional acreditado, tras llegar a un acuerdo mínimo entre sus miembros y aceptar un marco ético de conversación.

De los enfoques clásicos de la terapia familiar sistémica podemos destacar algunas formas de abordaje:

La orientación estructural, tras la puesta en escena de las pautas disfuncionales, trabaja sobre el establecimiento de límites equilibrados entre los subsistemas familiares y la modificación de las construcciones cognitivas que perpetúan las relaciones conflictivas (5).

Los modelos estratégicos buscan provocar desafíos que lleven a la familia a contemplar el absurdo de su sistema de distribución del poder dentro del grupo y sustituirlo por un equilibrio aceptable (6).

Los modelos narrativos trabajan sobre la deconstrucción de la historia problemática de la familia mediante un proceso conversacional en el que se intenta explicitar lo no explicado, la vida familiar expresada fuera del problema, y la construcción de una historia alternativa que tiene en el presente terapéutico un origen (7).

Nuestra aportación a los modelos de organización del proceso terapéutico pretende diferenciar fases de trabajo específico por parte del equipo y métodos de análisis comunicacional concreto que faciliten y unifiquen el trabajo con las familias. 
ORIGINALES Y REVISIONES

Pretendemos hacer ver el proceso terapéutico como el camino que ha de andar la familia desde la configuración de sus relaciones, propias de un espacio privado limitado que les mantiene en un conflicto hiriente que les hace pedir ayuda, hasta una nueva configuración relacional más propia de los valores del espacio público en la que el reconocimiento y respeto mutuo les aporten mejores condiciones existenciales.

\section{Los espacios comunicacionales}

Partimos de la fenomenología de la vida activa propuesta por Hannah Arendt y sus distinciones entre la esfera pública y la privada, que desarrollamos apoyándonos en las aportaciones de Ernesto Garzón Valdés para la diferenciación de los distintos espacios relacionales y teniendo en cuenta, en la práctica terapéutica concreta, los niveles de legitimación de las instituciones sociales de Berger y Luckman aplicados a la familia por Salvador Minuchin.

$\mathrm{Si}$ de una manera gráfica pudiéramos delimitar el componente físico de estos espacios en el que se producen las relaciones humanas, la esfera de la intimidad se verificaría al cerrar la puerta de la alcoba y la esfera privada al cerrar la puerta del hogar que sería su limitante. La esfera social se verificaría en la calle pudiendo, las más de las veces, representar una extensión poblacional de los valores privados, quedando para el espacio público, aquel en el que la libertad del discurso y de la inserción de la identidad en el diálogo con los otros es posible.

El espacio privado aporta un mínimo de bienes y un lugar de pertenencia para fundamentar la dignidad personal y el único lugar oculto del mundo común público a donde los miembros puedan retirarse y proteger la intimidad. La palabra privado cobra de su original sentido privativo, su significado. Para Hannah Arendt vivir una vida privada por completo significa, por encima de todo, estar privado de cosas esenciales a una verdadera vida humana: estar privado de la realidad de ser visto y oído por los demás, estar privado de una objetiva relación con los otros que proviene de hallarse relacionado y separado de ellos a través del intermediario de un mundo de cosas, de intereses comunes; estar privado de insertar en el mundo la propia vida y comenzar una historia personal (8).

En la familia los otros está severamente restringido. Esta diferenciación de espacios relacionales no pretende ser maniquea sino funcional. La propia diferenciación entre estas esferas reclama una reconsideración ya que lo privado también alberga el lugar que toda existencia humana necesita para cuidar las bases de la vitalidad sin las que no habría realmente un quién que saliera al espacio público. La familia entendida como totalidad, necesita producir cierto tipo 
de consenso acerca del sentido y finalidad de sus vidas juntas y esta necesidad se gesta en las remodelaciones evolutivas que se producen en el espacio privado.

Garzón Valdés considera que lo íntimo es el ámbito de los pensamientos de cada cual, de la formación de decisiones, de las dudas que escapan a una clara formulación, de lo reprimido, de lo aún no expresado y que quizás nunca lo será. Dentro del ámbito de la intimidad caen también aquellas acciones cuya realización no requiere la intervención de terceros y tampoco les afecta. A la privacidad correspondería el ámbito donde pueden imperar exclusivamente los deseos y preferencias individuales reservado a un tipo de relaciones interpersonales en donde la selección de los participantes depende de la libre decisión de cada individuo. Lo público está caracterizado por la libre accesibilidad de los comportamientos y decisiones de las personas en sociedad.

Si lo íntimo estaba caracterizado por su total opacidad, lo que caracteriza a lo público es la transparencia. Entre estos dos extremos cabría ubicar el ámbito de lo privado como aquél en donde impera una transparencia relativa (9). En el ámbito de lo privado, aceptamos reglas de convivencia que, por una parte, tienden a preservar nuestra intimidad y, por otra, erigen barreras a la invasión de lo público.

Las líneas de demarcación de los distintos espacios en la estructura familiar se realizan mediante sistemas de legitimación que reposan en tradiciones y mitos y se expresan en el lenguaje. Tienden a la protección del entramado relacional de la familia y contienen esquemas explicativos simples que sirven a los miembros para dotar de significado a los hechos (5).

La terapia familiar tiene sentido en la medida en que las propias reglas activas dentro del espacio privado familiar están generando y perpetuando conflictividad y sufrimiento. Es más, amenazan la preservación de la intimidad y restringen la incorporación de los miembros a un espacio donde insertar su identidad, el público.

No obstante hemos comprobado cómo estos conceptos relacionales no deben ser identificados sólo con referentes espaciales físicos del tipo espacio privado igual a domus, espacio público igual a calle. Más bien pensamos que hablamos de espacios relacionales en tanto que lo que realmente los define son las dinámicas relacionales que en ellos se establecen, de forma que podemos observar espacios públicos en la privacidad doméstica, porque cumplen las características que les asignamos al espacio público y viceversa, observamos dinámicas relacionales que se corresponden con los espacios privados que tienen lugar en la vida pública; nuestra actualidad social y política ofrece numerosos ejemplos donde las características de la relación descritas para el espacio privado son fácilmente detectables para el observador medio.

En el apartado siguiente conceptualizamos las características del espacio privado familiar involucradas directamente en la conflictividad sin menosprecio 
ORIGINALES Y REVISIONES

de las potencialmente beneficiosas y necesarias que deben ser explicitadas durante el proceso de una terapia familiar.

\section{Conceptualización del espacio privado}

Identificamos en el espacio privado las siguientes características:

Asimétrico,

Jerarquizado,

Mitológico,

Normativo.

Asimétrico porque la distribución de autoridad está necesariamente concentrada en unas pocas figuras en orden a asegurar la subsistencia de la prole; jerarquizado en el sentido de que los roles determinan la posibilidad de ejercicio del poder y son difícilmente intercambiables; mitológico en el sentido que en su organización y normas internas, la distribución de roles e identidades toman sus significados de una narración simbólica que actúa como matriz de las orientaciones de sentido de una tradición transgeneracional, y normativo porque está definido por reglas tanto explícitas como implícitas que provocan la recurrencia de pautas conductuales (10).

\section{Demarcación conceptual del espacio público}

El espacio público por su parte debe presentar las siguientes características para identificarlo:

Igualitario,

Democrático,

Razonable,

Ético.

Igualitario pues posibilita la expresión de la pluralidad humana con su doble carácter de igualdad y distinción mediante un equilibrio en el montante de poder que cada miembro necesita para asegurar su propia representatividad en el discurso conjunto de la familia. Ser visto y oído por otros deriva su significado del hecho de que todos ven y oyen desde una posición diferente (8); democrático porque basará sus representaciones, el imaginario que construye, sobre las aportaciones de todos los miembros que desarrollan el discurso, asegurando que nadie quede silenciado ni nadie se reserve información privilegiada. Razonable porque 
el imaginario que se construye debe ser argumentado en aportaciones llenas de sentido común, es decir, percibidas por todos los participantes, y ético porque las acciones deben ser justificadas sobre la base de evitar el sufrimiento.

\section{Lo que hay en juego}

En la acción de escuchar se rompe la reiteración del diálogo yo-mí con la solicitud de ser escuchado porque la reflexividad lleva en sí misma la amenaza de replegarse sobre sí, en lugar de una apertura hacia el establecimiento de la identidad.

El sentido común entraña la característica de poder pensar particularmente con los otros y desde la perspectiva de otros dando una sensación de realidad y de estabilidad al cambiante mundo de las relaciones humanas. Esto no solo se debe a que el ser humano sea un ser pensante, sino también a que existe sólo en lo común propio de la pluralidad, de modo que su razón demanda comunicación y podría extraviarse si no lo hace, pues como observó Kant, la razón no se adapta al aislamiento sino a la comunicación.

La traslación de un espacio a otro, que implica el inicio de una relación ayuda, se enfrenta decididamente con la patología derivada de la puntuación de la secuencia de hechos que se fundamenta en el uso inadecuado del poder y en la privación del acceso a la información (11). Puntuar equivale a establecer el valor o el carácter de la relación desde una situación de legalidad: pretende imponer el sentido y el juicio de valor de las conductas. Practica una apertura en lo real que va más allá de lo dado, privilegiando a alguien o a un grupo, que se pone aparte con la intención de convertirse en absoluto y dilucidar el sentido último de todo lo que acaece. En otras palabras, la puntuación privilegia juicios y personas sobre otras perspectivas y seres. Se apoya en la utilización incorrecta de los privilegios del espacio privado de relación. En la familia remite a un ejercicio inadecuado de la conyugalidad o la parentalidad cuyas consecuencias quedan fuera del control de quién actúa introduciendo un elemento adicional de arbitrariedad.

Cada mensaje tiene valores informativos y valores conativos o de control. Estos últimos pretenden definir la relación. La puntuación pretende quitar a algunos su propia voz aunque paradójicamente necesita de ellos para afirmar su dominio. La comunicación derivada de un marco de puntuación opresivo está limitada a lo permisible en una estructura relacional en la que a algunos les es permitido hablar, mientras otros pueden hablar dentro de los límites impuestos por los primeros. La psicoterapia viene a suplir la comunicación no efectuada en la cotidianidad (12). Es una invitación a pensar, particularmente a pensar desde el punto de vista de otros. 
ORIGINALES Y REVISIONES

En las distintas modalidades de abordaje de la problemática familiar podemos distinguir la presencia de este intento de migración: los terapeutas pretenden confrontar las dificultades familiares contrastándolas, mediante técnicas de comunicación, con las nuevas posibilidades de relación que podrían experimentarse si se incorporan valores del espacio público a la convivencia familiar. El proceso de incorporación de estos valores es variable según los autores.

Castilla del Pino señala que uno de los efectos de la incomunicación es el desarrollo en el aislamiento, en la individualidad, y, en consecuencia, sin el refrendo por parte de los demás de la viabilidad del proyecto que para sí mismo se traza o del pensamiento obtenido sobre una realidad (12). Esto implica que la introspección, el interés de la conciencia por su propio contenido, no puede construir una identidad ya que está limitada a un mundo construido por la propia mente sin validación.

En nuestro caso, el equipo toma sobre sí la responsabilidad de representar al mundo, al espacio público con sus valores. La terapia será una reiteración recuperadora y confrontadora que pretende que salga a la luz lo no dicho explícitamente y supuesto en todo pensamiento o conducta. Busca modificar la puntuación hacia otros significados que alivien sufrimientos. En otras palabras, dar voz al sufriente.

El sentido común que defendemos es el relacional, la participación en un mundo común. La terapia enfrenta a la familia con la necesidad de recuperar el sentido común, de enfrentar su distorsión del mundo, con la intromisión de parte del mundo, el espacio público, que representa el equipo terapéutico.

¿Cómo se efectúa esta migración entre espacios?

Distinguimos tres etapas guía por las que transita la familia durante la terapia. Su reconocimiento determina las actividades del equipo en cada fase y proporciona indicadores relacionados con el alta clínica o la recomendación de seguimiento por los terapeutas individuales que hayan realizado la derivación. Estos son:

\section{La familia como comunidad asociativa}

2. De la comunidad asociativa a la comunidad colaborativa

3. De la comunidad colaborativa a la comunidad comunicativa

\section{La familia como comunidad asociativa}

El rasgo distintivo de la familia como comunidad asociativa es que en esta esfera doméstica sus miembros viven juntos llevados por sus necesidades y exi- 
gencias para su mantenimiento individual y supervivencia; necesitan para ello la compañía de los demás y ejercer un sistema de interdependencia y gobierno.

La familia en esta configuración relacional se encuentra en medio del conflicto y de sus intentos de solución siendo esta fase una etapa lenta en la que los terapeutas sabemos que es muy difícil, cuando no imposible que sus miembros miren más allá del propio sufrimiento, de la propia defensa individual, de la propia puntuación de los hechos y del uso particular de las características limitantes del espacio privado. Esta situación favorece el desarrollo de jerarquías basadas en la fuerza, asimetrías paradójicas con un núcleo de importante contradicción, normas sin autoridad moral y uso de la mitología como recurso amenazante de exclusión, en lugar de su percepción como el esfuerzo interpretativo de las generaciones precedentes. La familia en estos momentos se comporta como una comunidad estratégica donde cada individuo es incapaz de hacer concesiones, pues aún espera lograr la imposición de su propia puntuación de hechos, el éxito de su cosmovisión. Sin embargo aceptan problematizar mediante el discurso sus pretensiones, de modo que el dialogo se presenta como un medio para alcanzar sus intereses personales, es decir como un instrumento de poder en lugar de una herramienta para la comprensión. La lucha reiterada por la imposición de perspectivas y el sufrimiento y desgaste de ella derivados, constituyen un embrión de la necesidad comunicacional entre los miembros de la familia y expresa la vinculación íntima que los une. La intensidad de la lucha es proporcional a la falta de reconocimiento que tienen los miembros entre sí. Desde una óptica hermenéutica estas tensiones reflejan las dificultades para incorporar la experiencia diversa de los miembros dentro de un patrón de relaciones que se ha quedado insuficiente y que busca a través del conflicto nuevas posibilidades.

Familia T. El hijo mayor vive en una situación de alejamiento con la amenaza de exclusión del resto de la familia formada por la madre y dos hijas más. La mitología que posibilita esta acción a la madre toma su poder de la narración que ella misma hace de su propia vida: sola se ha enfrentado a numerosas adversidades y no ha sido carga para nadie. Establece la norma de que a partir de cierta edad se es independiente o se es un aprovechado que debe ser expulsado. Las hijas menores ocupan un lugar preferente en la jerarquía al no haber tenido que demostrar aún su competencia para cumplir la norma. La asimetría determina que las razones del hijo para explicar sus dificultades no sean tenidas en cuenta.

\section{Contenidos de la tarea terapéutica}

1. El objetivo será el contrato, acuerdo entre voluntades individuales bajo la premisa de preeminencia del egoísmo. La explicitación de estos pactos no pasa de la expresión de la ganancia particular para cada miembro quedando en un segundo plano el beneficio familiar. 
ORIGINALES Y REVISIONES

2. El contrato es una idea de indudable utilidad práctica, implica una obligación de acatamiento individual para conquistar la paz familiar como forma de bien común. Intenta poner las bases para alcanzar una situación favorable a la comunicación racional.

\section{Contratos más habituales}

1. La firma del contrato terapéutico. El contrato que nuestro equipo propone define principios de confidencialidad, solicitud de permisos, da primeros pasos en la participación colectiva en la consulta, afronta la disipación de información oculta y crea compromisos temporales para dar oportunidad a dialogar suficientemente sobre todo.

Nuestro contrato terapéutico (extractos): Cumplimentando esta solicitud de tratamiento, firmada por los miembros de la familia, quedan ustedes anotados en la lista de espera del Programa de Terapia Familiar Sistémica... La cumplimentación y firma de esta solicitud supone una aceptación de las características del trabajo clínico antes señaladas... La información escrita por ustedes en esta solicitud debe reflejar las opiniones de común acuerdo, para lo que sugerimos se reúnan todos los miembros de la familia para contestarla... ¿Todos los miembros de la familia conocen la totalidad del contenido de este documento? Especificar quién sí y quién no... Fecha, firma, nombre y apellidos de los miembros de la familia.

2. El pacto por la vida: deponer la amenaza de autolisis para mantener la cohesión familiar. Limitar la escalada simétrica cuya máxima expresión en este momento es la apuesta de la propia vida para doblegar las voluntades de los miembros y garantizar la estabilidad del estatus sistémico alcanzado.

3. El pacto contra la violencia: toda violencia se opone a la terapia, el dominio por el terror impide la libre comunicación. Aquí la escalada simétrica llega a su máxima expresión apostando la integridad del otro para mantener la cohesión del grupo.

4. El pacto para el uso de la palabra durante el encuentro. El respeto a los tiempos de expresión, la valoración equivalente de las perspectivas, y la concisión son elementos que comienzan a demarcar el respeto a la autonomía de cada participante en el encuentro clínico.

5. Pacto para realización de tareas terapéuticas. Las tareas son ejercicios conductuales de entrenamiento en el ámbito extra-clínico que suponen un compromiso de los distintos miembros para hacer cosas diferentes a las que hasta ahora han sido recurrentes y agotadoras. 


\section{La familia como comunidad colaborativa}

La distinguimos como la familia bajo la contención de los contratos terapéuticos. Este logro representa el primer y fundamental paso de la familia hacia metas comunes más allá de la percepción de beneficios individuales: Si en la primera etapa los miembros de la familia escuchaban más al equipo, ahora comienzan a escucharse entre ellos mismos.

Por tanto, el objetivo del equipo es la comunicación en la familia. La percepción de dificultades en la comunicación, no sentirse visto u oído o entendido, más que no ver, oír o entender a los otros miembros, es una constante en la descripción de los motivos de consulta que manifiestan las familias al rellenar la solicitud de tratamiento. El equipo debe ser experto en comunicación, en la familia los expertos son los propios miembros de la familia (7). Asumimos las propuestas habermasianas de la pragmática universal para la que la comprensión por medio del lenguaje representa el caso más básico de acción comunicativa. El equipo pone su técnica al servicio de la razón comunicativa en el convencimiento ingenuo de que existe una necesidad de intercomunicación orientada a la emancipación humana en todos los miembros y que esta necesidad confiere un cierto grado de reflexividad sobre la propia conducta lingüística y la de sus interlocutores (13). La idea subyacente es que los miembros de la familia saben cómo llevar a cabo realizaciones comunicativas satisfactorias sin ser capaces de dar una explicación explícita de los criterios en que se basan, si bien son capaces de reconocerlos cuando los ponen en práctica.

\section{Contenidos de la tarea terapéutica}

El equipo revisa y depura las pretensiones de validez de cada afirmación en un ejercicio de claridad comunicacional donde vayan emergiendo las personas y retrocediendo los discursos. Procura en todo momento señalar el potencial emancipatorio incluido en las diferentes intervenciones que realizan los miembros especialmente en las paradójicas porque al encerrar una contradicción de términos facilita el análisis dialéctico.

En estos momentos la familia es una comunidad colaborativa con el equipo. El equipo terapéutico pretende que todos puedan hablar de todo y para ello se guía por la aplicación de las tareas propias de la pragmática. Siguiendo a Habermas, la tarea del equipo terapéutico consiste en identificar y construir con la familia los presupuestos generales de la acción comunicativa orientada a alcanzar el entendimiento intersubjetivo. El fin de una comunicación es la provocación de un estar de acuerdo que termina en la comunión intersubjetiva de la comprensión recíproca, del saber participado y de la confianza recíproca (14).

El equipo enmarca la conversación bajo dos principios conversacionales que Paul Grice definiera de una forma sencilla y efectiva (15): 
ORIGINALES Y REVISIONES

- El principio cooperativo: los participantes esperan que cada uno hará su contribución conversacional tal como es requerida, en el escenario en que ocurre, para el propósito o dirección aceptado en el intercambio verbal.

- El principio informativo: el destinatario tiene la licencia para aplicar su conocimiento del mundo para inferir un sentido implícito que es más intensamente informativo que la actual expresión.

La obra de Grice aporta, a nuestro entender, una consideración de eficacia a la comunicación de modo que sea exitosa para las personas implicadas.

Familia B. Formada por padre, madre y dos hijas que viven con sus parejas pero que se encuentra atrapadas en su familia de origen donde expresan manifestaciones de angustia, somatizaciones y preocupación por sus padres.

- Madre: hay cosas que sé pero que me reservo para no dañar a mi familia (Aparentemente transgrede el principio cooperativo. Es paradójico, pues si es un secreto no hablaría de su existencia y como lo hace es su contribución conversacional de modo que realmente sí quiere hablar de ello)

- Terapeuta: entendemos que quiera cuidar a su familia llevando sola esta información. No obstante sabemos que las personas podemos cuidarnos mejor si compartimos los pesos que llevamos dentro con los demás. De este modo nos informamos a través de los que nos cuidan y no por personas ajenas que no tengan este interés por nosotros. (Amparándose en el principio informativo intenta desactivar los secretos que se presentan como información privilegiada; instrumento éste del dominio relacional al condicionar la puntuación de los hechos)

El marco proporcionado por estos dos principios, nos permite trabajar directamente con las pretensiones de validez propuestas por Habermas aplicadas a las unidades comunicacionales que cada miembro de la familia comparta con los demás. El equipo estará atento a que estas se cumplan, proporcionando la base del ejercicio comunicativo de las sesiones. Su cumplimiento da cuenta y ejercita las competencias comunicacionales fundamentales: distinguir entre un mundo público (lo que realmente es) y uno privado (lo que parece ser); distinguir entre el sí mismo individualizado y sus distintas expresiones y acciones en las que se manifiesta; $y$, distinguir en la reciprocidad de expectativas interpersonales entre lo que es y lo que debe ser (14).

Las pretensiones de validez que propone Habermas y que el equipo tiene en cuenta en la moderación del diálogo entre los miembros de la familia son las siguientes:

1. Inteligibilidad: expresiones comprensibles para todos

2. Verdad: coherencia con la historia construida 
3. Veracidad: genuinas y sinceras

4. Rectitud: tender a un acuerdo recíproco sobre la base de normas y valores considerados como válidos.

El estar de acuerdo reposa sobre la base del reconocimiento de las respectivas pretensiones de validez: comprensibilidad, verdad, sinceridad y justicia en relación a normas y valores. El que habla debe elegir una proposición comprensible, para que el que habla y el que escucha puedan entenderse el uno al otro; el que habla debe tener la intención de comunicar un contenido proposicional verdadero, para que el que escucha pueda compartir su saber; el que habla debe querer exteriorizar las propias intenciones de modo verdadero, para que el que escucha pueda tener confianza en aquello que dice; el que habla debe finalmente buscar la expresión justa en la consideración de normas y valores vigentes, para que el que escucha la pueda aceptar de modo que ambos, el que habla y el que escucha, puedan ponerse de acuerdo en orden a un fundamento normativo reconocido (14).

El fracaso en alcanzar estas pretensiones dentro de la narración se intenta resolver habitualmente mediante dos salidas que perpetúan la conflictividad y que el equipo debe reconocer para no involucrase en ellas:

- Recurso a la acción estratégica orientada al éxito en la que se podría utilizar cualquier medio de seducción incluida la fuerza.

- Traslado de la comunicación al nivel del discurso donde es problematizada y llevada a la búsqueda cooperativa de argumentos.

Durante el curso de la terapia, el terapeuta debe asegurar que todo lo expresado sea inteligible de modo que cada avance se incorpore como material de un pacto de lectura: todos entendemos que estamos hablando de lo mismo. La verosimilitud se desarrolla en el modo de la coherencia de la narración. El terapeuta la utilizará como la herramienta principal de la racionalización del discurso. Por último la rectitud hace referencia a la necesidad de un ajuste ético o moral del discurso de modo que todo lo que produzca sufrimiento debe ser explicitado y enjuiciado de cara a su afrontamiento. El abandono del sufrimiento tiene que ser sentido como una conquista de una libertad afirmativa en la que sus condiciones de realización son la auto-realización y el reconocimiento intersubjetivo. De este modo la verdad y la rectitud constituyen las pretensiones más relevantes para la racionalidad comunicativa.

Familia $H$ ( $6^{a}$ sesión). Formada por la madre y dos hijas adultas jóvenes no emancipadas. Acuden a la Unidad tras una larga historia de sufrimiento y conflictividad que les ha llevado a la imposibilidad de hablarse y escucharse sin 
ORIGINALES Y REVISIONES

sentir el riesgo de la ruptura. La pareja parental estaba dañada desde sus inicios. El embarazo y posterior aborto de la hija mayor fue utilizado por la pareja para poner fin a su convivencia, trasladando la responsabilidad de su fracaso como pareja, a la entonces adolescente. La madre penaliza durante dos años a esta hija con descalificaciones y culpa. Cuando es confrontada por el relato de las hijas aduce una amnesia selectiva durante esos años.

- Madre. No recuerdo nada de lo que ellas me dicen. Estaba tan deprimida entonces y además bebía. Ahora me siento mal porque ellas me ven como culpable.

- Hija. Me arrepiento de haber comentado aquí todas estas cosas porque el ambiente en casa es peor. Antes lo llevaba yo sola pero ahora ella se hace una víctima.

El cierre que propone la madre transgrede las pretensiones de verdad, veracidad y corrección. No se adecúa a los recuerdos de las dos hijas y de una hermana suya; no es aceptable la autenticidad de la amnesia que además resiste a la actualización realizada por el relato; no se presenta bajo el valor de responsabilidad, de asunción del daño y solicitud de perdón. La hija pone en su persona lo que espera de la madre, arrepentimiento. El equipo orienta la conversación hacia otras posibilidades ya que la comunicación en estos términos es un fracaso e imposibilita un consenso emocional de reconciliación.

\section{La familia como comunidad comunicativa}

Entendemos la comunicación sanadora como la experiencia de reciprocidad entre sujetos en la que cada miembro reconoce a los otros como agentes morales portadores de razones y concede el derecho a la igualdad y libertad. Cuando los miembros de la familia experimentan y expresan esta vivencia tenemos la razonable certidumbre de que han alcanzado esta etapa de crecimiento personal.

El diálogo es un fin en sí mismo ya que lo que se comunica son formas de vida. Sujetos capaces de interpretar y dar sentido a la realidad.

El objetivo es el reconocimiento. El reconocimiento de sí por parte de la persona actuante y sufriente se caracteriza como saberse capaz de ciertas realizaciones.

\section{Contenidos de la tarea terapéutica}

La posición activa del terapeuta estriba en su comportamiento racional con el que puede reaccionar a las cualidades valiosas de una persona y saber explicitar esa capacidad de acción en el encuentro clínico mediante una perspicaz percepción de los valores de cada uno. El terapeuta asume la representación de una continuidad simbólica de la comunidad humana que concede el reconocimiento negado en la experiencia del paciente. El reconocimiento en terapia familiar tiene 
una dificultad añadida que no parece existir en la terapia individual. En esta la función simbólica del terapeuta es determinante, pero en aquella, la simbología se difumina ante el papel real de los miembros de la familia que son los que tienen que conceder el reconocimiento. El terapeuta representa a una comunidad mediadora, el espacio público, pero la familia es la comunidad reconocedora. No puede ser sustituida por el terapeuta porque donde hay presencia real, la simbología toma otras dimensiones secundarias.

El reconocimiento restituye a la familia su capacidad de cuidar como un complejo de actitudes y disposiciones, de saberes y competencias, cuya relevancia, puesta de manifiesto en el espacio público, vuelve a la privacidad e intimidad con la impronta del valor democrático (16). Conduce al establecimiento de relaciones privadas éticas en las que la reconstrucción de las condiciones de comunicación dañadas y la resistencia práctica del sujeto moralmente dañado abocan a una situación renovada de reconocimiento recíproco (17).

Familia H (Última sesión):

- Madre a la hija: Lo siento. Perdóname por los malos momentos. Quiero ser un punto fuerte de apoyo en tu vida. Eres mi vida. El pasado, pasado está. Confío en ti y quiero que confíes en mí. No te voy a volver a decepcionar. Viviremos con un optimismo que merezca la pena. No volveremos a pasar por ahí. Procuraré recompensarte.

- Hija a la madre: (Le entrega un collage con escenas de las dos desde pequeña) Está todo olvidado. Confío en ti. Por encima de todo te quiero. Eres mi madre, la que me ayuda, sufre y siempre está ahí. Te pido perdón porque no te he facilitado el camino.

- Hijas entre sí: Te quiero mucho. Eres la primera persona en quién confío y tú lo sabes.

El fin de la terapia se anuncia. Cuando los miembros de la familia alcanzan este nivel, el diálogo es el medio para reconocer y su posibilidad libre es el reconocimiento mismo. Hasta la polémica se incorpora al diálogo pues encierra la promesa de un reconocimiento. La razón carece de entidad dictatorial, depende del consenso de ciudadanos libres.

El ejemplo anterior presenta una familia que transitó por las distintas fases hasta un alta consensuada por alcanzar los objetivos, plenos en este caso. No siempre es así. En nuestros registros solo un tercio de las familias llegan a un alta consensuada con el equipo debido al logro de los objetivos terapéuticos comunes. Algunas familias prefieren permanecer en un estadío colaborativo modulado por algunos pactos de supervivencia y otras, lamentablemente, prefieren permanecer en una posición conflictiva cronificada. 
ORIGINALES Y REVISIONES

\section{Conclusiones}

La terapia familiar se enriquece y orienta teniendo en cuenta la interacción entre los espacios relacionales y la conflictividad que surge de la transgresión de fronteras o de la falta de permeabilidad a los valores del espacio público. Atendiendo a las posibilidades de la familia en cada fase del proceso terapéutico, los terapeutas podrán centrar sus actuaciones sin tensar inapropiadamente el momento evolutivo del que la familia es capaz. Los valores del espacio público se pueden aplicar de forma práctica siguiendo las propuestas de la pragmática universal de Habermas y las nociones de la teoría del reconocimiento.

\section{BIBLIOGRAFÍA}

(1) Boxó Cifuentes JR. La acción de escuchar. Med fam Andal 2010; 2: 178-183

(2) Benito Riesco, O.; Rubio González, M.A.; Boxó Cifuentes, J.R.; Ruiz Sicilia, L.; Aragón Ortega, J. Family Profiles and Activities of a Public Unit Family Therapy. WPA Thematic Conference. Granada 2012.

(3) Boxó Cifuentes JR, Aragón Ortega J, Ruiz Sicilia L, Benito Riesco O, Rubio González MA. Análisis de la connotación positiva como promotor lingüístico de cambios. Mosaico 2013; 54: 16-22.

(4) Boxó Cifuentes JR, Aragón Ortega J, Ruiz Sicilia L, Benito Riesco O, Rubio González MA. Teoría del reconocimiento: aportaciones a la psicoterapia. Rev. Asoc. Esp. Neuropsiq., 2013; 33 (117): 67-79.

(5) Minuchin S y Fishman HC. Técnicas de terapia familiar. Paidós Terapia Familiar. Barcelona 1997.

(6) Navarro Góngora J. Técnicas y programas en terapia familiar. Paidós Terapia Familiar. Barcelona 1996.

(7) Ramos Gutiérrez R. Narrativas contadas, narraciones vividas. Paidós, Barcelona 2001.

(8) Arendt H. La condición humana. Paidós. Barcelona 2005.

(9) Garzón Valdés E. Lo íntimo, lo privado y lo público. Cuadernos de transparencia. IFAI, México 2005.

(10) Watzlawick P, Beavin Bavelas L y Jackson DD. Teoría de la comunicación humana. Herder Barcelona 1997.

(11) De la Mata Impuesto JL. http://www.joseluisdelamata.com/IZARGAIN-textos/Axiomas\%20de\%20la\%20Pragmatica.pdf

(12) Castilla del Pino C. La incomunicación. Península. Barcelona 1981.

(13) Corbí J, Habermas J. Pragmática universal y normatividad. Daimon, 1989; 1: 39-57.

(14) McCarthy Th. La teoría crítica de Jürgen Habermas. Tecnos, Madrid 1987.

(15) Herrero Sicilia J. Teorías de pragmática, de lingüística textual y de análisis del discurso. Ediciones de la Universidad de Castilla La Mancha. Cuenca 2006. 
ORIGINALES Y REVISIONES

(16) Muñoz Terrón JM. Cuidar del mundo, Labor, trabajo y acción en una compleja red de sostenimiento de la vida. Isegoría 2012 (47): 461-480.

(17) Honneth A. Crítica del poder. A. Machado Libros, Madrid 2008. 